\title{
Effect of ranibizumab on serous and vascular pigment epithelial detachments associated with exudative age-related macular degeneration
}

This article was published in the following Dove Press journal:

Drug Design, Development and Therapy

9 July 2013

Number of times this article has been viewed

\section{Georgios D Panos'}

Zisis Gatzioufas'

loannis K Petropoulos'

Doukas Dardabounis ${ }^{2}$

Gabriele Thumann'

Farhad Hafezi'

'Department of Ophthalmology, Geneva University Hospitals and Faculty of Medicine of the University of Geneva, Switzerland; ${ }^{2}$ Department of Ophthalmology, University Hospital of Alexandroupolis, Greece
Correspondence: Georgios D Panos Department of Ophthalmology, Geneva University Hospitals, Rue Alcide - Jentzer 22, I2I I, Geneva 14, Switzerland

Tel +4I 795534739

Fax +4I 223828382

Email georgios.panos@hcuge.ch
Purpose: To report the effect of intravitreal ranibizumab therapy for serous and vascular pigment epithelial detachments (PED) associated with choroidal neovascularisation (CNV) secondary to age-related macular degeneration (AMD).

Methods: In a prospective study, best-corrected visual acuity (BCVA) and optical coherence tomography (OCT) data were collected for 62 eyes of 62 patients, with serous or vascular PED associated with CNV secondary to AMD. Intravitreal ranibizumab $0.5 \mathrm{mg}$ was administered with a loading phase of three consecutive monthly injections, followed by monthly review with further treatment, as indicated according to the retreatment criteria of the PrONTO study. The change in visual acuity and PED height from baseline to month 12 after the first injection was determined.

Results: Sixty-one eyes of 61 patients (one of the patients developed retinal pigment epithelial tear and was excluded from the study) were assessed at the 12-month follow-up examination. There were two types of PED, including vascular PED in 32 patients (Group A) and serous PED (Group B) in 29 patients. The mean improvement of mean BCVA from baseline to 12 months was $0.09 \log$ MAR (Logarithm of the Minimum Angle of Resolution) in Group A and 0.13 logMAR in Group B. Both groups showed significant improvement of the mean BCVA 12 months after the first injection compared with the baseline value $(P<0.05)$. In relation to the PED height, the mean decrease of mean PED height from baseline to 12 months was $135 \mu \mathrm{m}$ in Group A and $180 \mu \mathrm{m}$ in Group B. Both groups showed significant reduction of the PED height during the follow-up period $(P<0.01)$. The PED anatomical response to ranibizumab was not correlated with the BCVA improvement in any of the groups. Apart from one patient who developed pigment epithelial tear no other complications were documented.

Conclusion: Ranibizumab is an effective and safe treatment for improving vision in patients with serous and vascular PED, although the anatomical response of the PED to ranibizumab may not correlate directly with the visual outcome.

Keywords: age-related macular degeneration, choroidal neovascularisation, intravitreal injection, pigment epithelial detachment, ranibizumab

\section{Introduction}

Age-related macular degeneration (AMD) is a leading cause of blindness in industrialised countries. ${ }^{1}$ AMD has been classified into a non-exudative or dry form and an exudative or wet form with choroidal neovascularization (CNV). Both of these forms lead to vision loss, but prognosis is even worse in patients affected by the exudative form, due to intra- and subretinal leakage and fibrotic lesions in the posterior pole. $^{2}$ 
Some patients with AMD may manifest retinal pigment epithelial detachment (PED), in which the retinal pigment epithelium (RPE) physically separates from the underlying Bruch's membrane. ${ }^{3}$ PEDs associated with AMD are classified by clinical and angiographic characteristics as drusenoid, serous, vascular or fibrovascular, and hemorrhagic. ${ }^{3-6}$

The management of patients with PED secondary to AMD is controversial due to the difficulty in specifying the presence of underlying $\mathrm{CNV}^{4}$ In addition, therapeutic options for PED are limited and usually provide poor outcome. ${ }^{7-12}$

Recently, agents inhibiting the activities of the vascular endothelial growth factor (VEGF), such as ranibizumab, bevacizumab and pegaptanib have been used for the treatment of CNV in patients with AMD. ${ }^{13-15}$

This prospective study reports the efficacy of intravitreal ranibizumab (Lucentis, Novartis, Basel, Switzerland) monotherapy as a treatment for serous and vascular PEDs associated with exudative AMD (CNV-PED).

\section{Material and methods}

This study was performed in accordance with institutional guidelines and the ethical standards of the Declaration of Helsinki. All patients were thoroughly informed and provided their consent to participate in the study.

In a prospective cohort study of 62 patients, baseline and follow-up data were collected for eyes with newly diagnosed serous and vascular PED associated with CNV secondary to AMD and treated with $0.5 \mathrm{mg}$ of intravitreal ranibizumab monotherapy. The study protocol included best-corrected Early Treatment Diabetic Retinopathy Study (ETDRS), visual acuity score (BCVA), PED height as measured by optical coherence tomography (OCT), and the assessment of intraretinal or subretinal fluid as evaluated by OCT.

Patients with BCVA worse than 20/400 at baseline, retinal angiomatous proliferation (RAP), polypoidal choroidal vasculopathy (PCV), PED without any evidence of CNV, haemorrhagic PED, mixed PED (co-existing fibrovascular and serous PED), co-existing ocular diseases such as glaucoma or diabetic retinopathy, and patients previously treated for AMD were not included in the study.

All patients were scanned with Spectralis OCT (Heidelberg, Germany). The built-in manual calliper tool was used to measure the PED height on the OCT monitor. Before treatment, fluorescein and indocyanine green (ICG) angiography (Spectralis, Heidelberg Engineering, Heidelberg, Germany) was performed to determine the baseline lesion features, and the images were evaluated by a single physician. According to baseline fluorescein and ICG angiography and OCT examination findings, PEDs were classified into two subgroups, vascular/ fibrovascular PEDs and serous PEDs as described by Pepple and Mruthyunjaya. ${ }^{4}$ The objective of classifying PED into two groups was to investigate whether vascular PED shows better or worse response to intravitreal ranibizumab than serous PED.

Routine examination included ETDRS BCVA, Goldmann applanation tonometry, slit-lamp biomicroscopy, fundoscopy, and OCT. Time points of measurements were prior to intravitreal ranibizumab treatment (baseline), after 3 months, after 6 months, and after 12 months from the first intravitreal injection. Moreover, fluorescein and ICG angiography was performed every 3 months after the first injection in order to determine new area of CNV.

Initial treatment was administered as a loading phase of three consecutive monthly $0.5 \mathrm{mg} / 0.05 \mathrm{~mL}$ ranibizumab injections, followed by a maintenance phase of repeated injections as required, according to visual acuity, OCT, and clinical examination findings. Intravitreal injections were repeated if any of the following changes were observed by the evaluating ophthalmologist: (1) Visual acuity loss of at least 5 letters with OCT evidence of fluid in the macula, (2) an increase in OCT central foveal thickness of at least $100 \mu \mathrm{m}$, (3) new macular hemorrhage, (4) new area of CNV, or (5) evidence of persistent fluid on OCT one month after the previous injection; according to the retreatment criteria of the Prospective OCT imaging of Patients with Neovascular AMD Treated with intraOcular Ranibizumab (PrONTO) study. ${ }^{16}$ The presence of PED alone was not considered to be indication for retreatment.

All data were collected on an MS-Excel 2003 spreadsheet (Microsoft Corporation, Redmond, 2003). The BCVA (Snellen equivalent of the ETDRS score) was converted to logarithm of the minimum angle of resolution (logMAR) equivalents for statistical analysis. Changes in the BCVA and PED height between baseline and the follow-up examinations were evaluated using paired Student's $t$-test. Results are presented as the mean \pm standard deviation (SD). In addition, Pearson's correlation coefficient was used to evaluate a possible correlation between anatomical and functional results. In all comparisons, $P<0.05$ defined statistically significant differences.

\section{Results}

The study included a total of 62 eyes presenting with exudative AMD associated with CNV-PED. One of the patients developed RPE tear after the second injection and was excluded from the study.

Group A (vascular PED) consisted of 32 patients of 69 years of age on average ( 55 to 80 years). The right eye was affected in 12 cases. Group B (serous PED) consisted 
of 29 patients of 71 years of age on average (55 to 83). The right eye was affected in 12 cases. Baseline characteristics of the two groups are summarized in Table 1.

In Group A, mean baseline BCVA was 0.57 $\log$ MAR. At month 3, month 6 and month 12 mean BCVA improvement was $0.06 \log$ MAR, $0.06 \log$ MAR and 0.09 $\log$ MAR respectively. No statistically significant difference was observed between baseline BCVA and 3 months BVCA $(P=0.12)$, or between baseline BCVA and 6 months BCVA $(P=0.14)$, however significant difference was documented between baseline BCVA and 12 months BCVA $(P=0.04)$.

In Group B, mean baseline BCVA was $0.60 \log$ MAR. At month 3, 6 and 12 mean BCVA improvement was 0.08 $\log$ MAR, $0.10 \log$ MAR and $0.13 \log$ MAR respectively. There was no statistically significant difference between baseline BCVA and 3 months BVCA $(P=0.06)$, or between baseline BCVA and 6 months BCVA $(P=0.09)$, however significant difference was observed between baseline BCVA and 12 months BCVA $(P=0.04)$.

The mean improvement of mean BCVA from baseline to 12 months was statistically significant for both the vascular PED group $(P=0.04)$ and the serous PED group $(P=0.04)$, but the difference of the improvement between the two groups was not significant (Unpaired $t$-test, $P=0.6$ ).

The detailed status of mean BCVA in both groups is outlined in Table 2.

In Group A, mean baseline PED height was $420 \mu \mathrm{m}$. At month 3, month 6 and month 12 mean PED height decrease was $80 \mu \mathrm{m}, 103 \mu \mathrm{m}$ and $135 \mu \mathrm{m}$ respectively. Statistically significant difference was observed between baseline PED height and PED height at $3(P<0.01), 6(P<0.01)$ and 12 months $(P<0.01)$.

In Group B, mean baseline PED height was $524 \mu \mathrm{m}$. At month 3, month 6 and month 12 mean PED height decrease was $107 \mu \mathrm{m}, 147 \mu \mathrm{m}$ and $180 \mu \mathrm{m}$ respectively. Statistically significant difference was observed between baseline PED

Table I Patient demographics and baseline characteristics

\begin{tabular}{lcc}
\hline Characteristic & $\begin{array}{l}\text { Group A } \\
\text { (vascular PED) }\end{array}$ & $\begin{array}{l}\text { Group B } \\
\text { (serous PED) }\end{array}$ \\
\hline Sex & $17 \hat{\jmath}, 159$ & $17 \hat{\jmath}, 129$ \\
Eye affected & 12 OD, 20 OS & 12 OD, 17 OS \\
Mean age \pm SD (years) & $69 \pm 8$ & $71 \pm 8$ \\
Mean baseline BCVA \pm SD (logMAR) & $0.57 \pm 0.28$ & $0.60 \pm 0.30$ \\
Mean baseline PED height \pm SD $(\mu \mathrm{m})$ & $420 \pm 110$ & $524 \pm 127$ \\
Mean average injections \pm SD & $6.1 \pm 1.8$ & $7.1 \pm 2.2$ \\
\hline
\end{tabular}

Abbreviations: BCVA, best-corrected visual acuity; logMAR, logarithm of the minimum angle of resolution; OD, right eye; OS, left eye; PED, pigment epithelial detachment; SD, standard deviation.
Table 2 Mean BCVA (logMAR)

\begin{tabular}{lllll}
\hline Group & $\begin{array}{l}\text { Baseline } \\
\text { Mean } \pm \text { SD }\end{array}$ & $\begin{array}{l}\text { 3 months } \\
\text { Mean } \pm \text { SD }\end{array}$ & $\begin{array}{l}\text { 6 months } \\
\text { Mean } \pm \text { SD }\end{array}$ & $\begin{array}{l}\text { I } 2 \text { months } \\
\text { Mean } \pm \text { SD }\end{array}$ \\
\hline Group A & $0.57 \pm 0.28$ & $0.51 \pm 0.30$ & $0.51 \pm 0.31$ & $0.48 \pm 0.32$ \\
Group B & $0.60 \pm 0.30$ & $0.52 \pm 0.28$ & $0.50 \pm 0.28$ & $0.47 \pm 0.28$ \\
\hline
\end{tabular}

Abbreviations: BCVA, best-corrected visual acuity; logMAR, logarithm of the minimum angle of resolution; SD, standard deviation.

height and PED height at $3(P=0.01), 6(P<0.01)$ and 12 months $(P<0.01)$.

The mean decrease of mean PED height from baseline to 12 months was statistically significant for both the vascular PED group $(P<0.01)$ and the serous PED group $(P<0.01)$, but the difference of the decrease between the two groups was not significant (Unpaired $t$-test, $P=0.097$ ).

The detailed status of mean PED height in both groups is depicted in Table 3.

This study did not reveal any correlation between change in the PED height and change in the BCVA either in Group A (vascular PED group) (Pearson's correlation coefficient, $r=-0.09 ; P=0.6$ ), or in Group B (serous PED group) (Pearson's correlation coefficient, $r=-0.04 ; P=0.8$ ).

Treatment was well tolerated, and there were no recorded cases of endophthalmitis, however one of the patients developed RPE tear after the second injection. This was the only complication.

Representative cases of PED evolution after intravitreal ranibizumab injection are depicted in Figures 1 and 2.

\section{Discussion}

The principal finding in this study is that ranibizumab is effective in improving vision in patients with CNV-PED secondary to exudative AMD, as evaluated at 12 months after the first intravitreal injection. At the end of the followup period, BCVA worsened only in five patients (15.6\%) in Group A, and in four patients (13.8\%) in Group B. According to previous studies, patients with PED, secondary to AMD, presented significant functional and anatomical benefit after anti-VEGF therapy compared with those receiving no treatment. ${ }^{17-19}$ However, the use of anti-VEGF agents for the treatment of PED associated with exudative AMD remains controversial. Introini et al in a retrospective study

Table 3 PED height $(\mu \mathrm{m})$

\begin{tabular}{lllll}
\hline Group & $\begin{array}{l}\text { Baseline } \\
\text { Mean } \pm \text { SD }\end{array}$ & $\begin{array}{l}\text { 3 months } \\
\text { Mean } \pm \text { SD }\end{array}$ & $\begin{array}{l}\text { 6 months } \\
\text { Mean } \pm \text { SD }\end{array}$ & $\begin{array}{l}\text { I } 2 \text { months } \\
\text { Mean } \pm \text { SD }\end{array}$ \\
\hline Group A & $420 \pm 110$ & $340 \pm 91$ & $317 \pm 97$ & $285 \pm 114$ \\
Group B & $524 \pm 127$ & $417 \pm 113$ & $377 \pm 111$ & $344 \pm 117$ \\
\hline
\end{tabular}

Abbreviations: PED, pigment epithelial detachment; SD, standard deviation. 


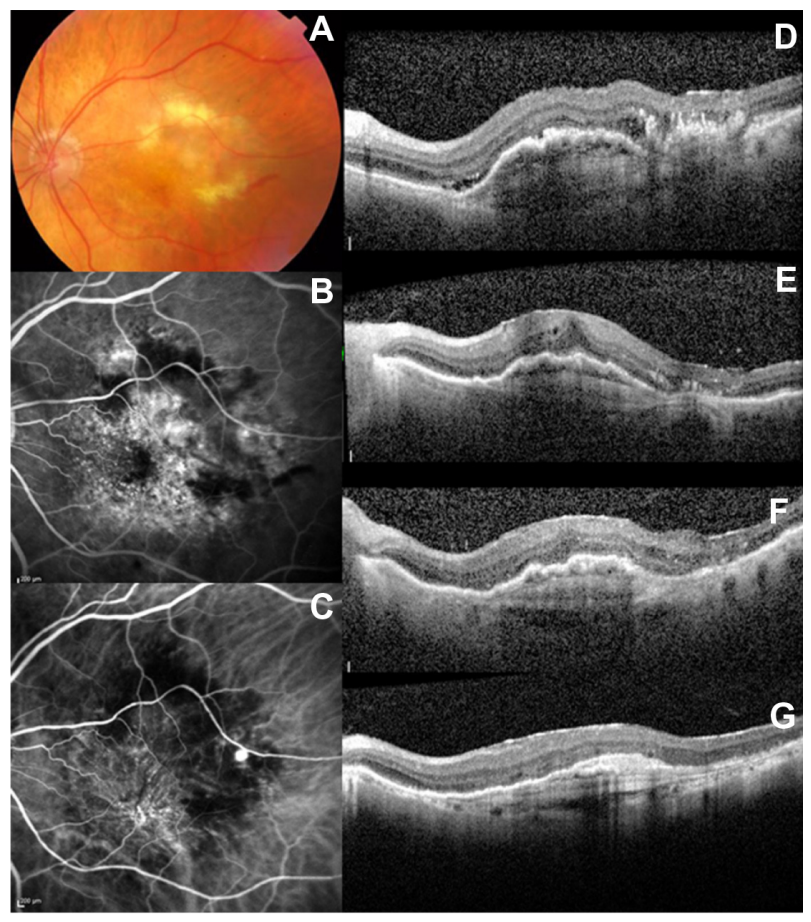

Figure I Selected case: A 76-year-old man was referred for decreased visual acuity in the left eye. Baseline examination, including (A) fundoscopy, (B) fluorescein angiography, (C) ICG angiography, and (D) macular OCT revealed a vascular PED associated with exudative AMD. The baseline BCVA and PED height were 20/80 and $380 \mu \mathrm{m}$, respectively. The same eye (E) at 3 months (BCVA: 20/70; PED height: 236 $\mu \mathrm{m}),(\mathbf{F})$ at 6 months (BCVA: 20/70; PED height: $176 \mu \mathrm{m}$ ), and (G) at 12 months (BCVA: 20/60; PED height: $166 \mu \mathrm{m}$ ), after the first ranibizumab injection.

Abbreviations: AMD, age-related macular degeneration; BCVA, best-corrected visual acuity; ICG, indocyanine green; OCT, optical coherence tomography; PED, pigment epithelial detachment.

of 132 eyes concluded that there is no effective therapy for PED secondary to AMD, suggesting that anti-VEGF therapy could achieve only stabilization of the disease, but with high risk of RPE tear. ${ }^{20}$ On the other hand, Arora and McKibbin in their retrospective study, including 19 eyes with vascularized and avascular PED, reported that moderate visual loss (15 or more ETDRS letters) was avoided in $95 \%$ of their patients and that $25 \%$ had a gain of 15 or more ETDRS letters, concluding that ranibuzumab is an effective treatment for both vascularized and avascular PED. ${ }^{21}$ In a prospective, comparative study, including 15 eyes, Arias reported that pegaptanib and bevacizumab are both an effective and safe treatment for CNV-PEDs secondary to AMD. ${ }^{18}$

Our study demonstrated that ranibizumab was effective in improving vision and macular anatomy in patients with both vascular and serous CNV-PED secondary to AMD. Regarding the number of injections required per eye, our study revealed no significant differences according to the type of PED ( $P=0.06)$ suggesting that equivalent suppression of $\mathrm{CNV}$ and resolution of exudative findings, such as subretinal or intraretinal fluid, were achieved after ranibizumab therapy

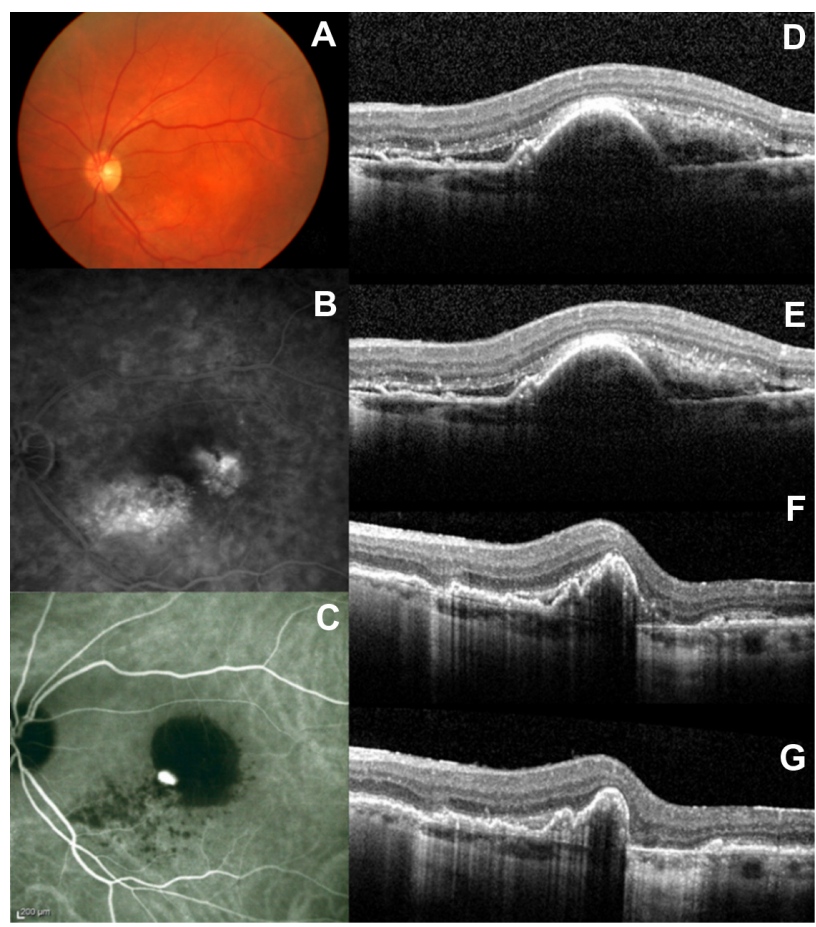

Figure 2 Selected case: A 72-year-old man was referred for decreased visual acuity in the left eye. Baseline examination, including (A) fundoscopy, (B) fluorescein angiography, (C) ICG angiography, and (D) macular OCT revealed a serous PED associated with exudative AMD. The baseline BCVA and PED height were 20/100 and $385 \mu \mathrm{m}$ respectively. The same eye (E) at 3 months (BCVA: 20/80; PED height: $358 \mu \mathrm{m}),(\mathbf{F})$ at 6 months (BCVA: 20/70; PED height: $297 \mu \mathrm{m})$, and (G) at 12 months (BCVA: 20/60; PED height: 25I $\mu \mathrm{m}$ ) after the first ranibizumab injection.

Abbreviations: AMD, age-related macular degeneration; BCVA, best-corrected visual acuity; ICG, indocyanine green; OCT, optical coherence tomography; PED, pigment epithelial detachment.

irrespective of the PED type. Regarding the influence of the PED height on BCVA, the BCVA response to treatment with ranibizumab was not correlated with the degree of response of the PED height to the treatment. Previous studies have also reported absence of any correlation between visual improvement and resolution of the PED. ${ }^{17,19,22}$ Therefore, the visual acuity improvement after ranibizumab may be independent of the response of PED to ranibizumab.

In literature there is only one recent study, evaluating the prognosis of patients with neovascular AMD after intravitreal anti-VEGF treatment according to the type of PED, by Inoue et al. ${ }^{22}$ This study, consisting of 56 eyes (11 serous PED, 28 fibrovascular PED, 7 mixed PED, and 10 hemorrhagic PED) treated with intravitreal ranibizumab and evaluated over a period of 12 months after the initial injection, demonstrated that ranibizumab was an effective treatment for stabilizing or improving vision in patients with PED, but with better results in patients with serous PED. On the contrary, our study showed that patients with serous CNV-PED secondary to AMD treated with ranibizumab presented the same functional and anatomical results as patients with vascular 
PED. This disagreement between these two studies could be explained by the following facts: (1) in the study of Inoue et al there was a significant difference in the sample size between the two groups (28 fibrovascular PED, 11 serous PED) whereas in our study the two samples were symmetric (32 fibrovascular PED, 29 serous PED) and (2) there were different inclusion criteria between the two studies; patients with PCV were excluded in our study, whereas patients with PCV were included in the study of Inoue et al in the group of serous PED.

In conclusion, ranibizumab is an effective and safe treatment for stabilizing or improving vision in patients with serous and vascular PED, although the anatomical response of the PED to ranibizumab may not correlate directly with the visual outcome. Larger studies with longer follow-up periods are necessary in order to validate the results of this study.

\section{Disclosure}

The authors report no other conflicts of interest in this work.

\section{References}

1. Klein R, Klein BE, Jensen SC, Mares-Perlman JA, Cruickshanks KJ, Palta M. Age-related maculopathy in a multiracial United States population: the National Health and Nutrition Examination Survey III. Ophthalmology. 1999;106(6):1056-1065.

2. Klein R, Klein BE, Jensen SC, Meuer SM. The five-year incidence and progression of age-related maculopathy: the Beaver Dam Eye Study. Ophthalmology. 1997;104(1):7-21.

3. Zayit-Soudry S, Moroz I, Loewenstein A. Retinal pigment epithelial detachment. Surv Ophthalmol. 2007;52(3):227-243.

4. Pepple K, Mruthyunjaya P. Retinal pigment epithelial detachments in age-related macular degeneration: classification and therapeutic options. Semin Ophthalmol. 2011;26(3):198-208.

5. Poliner LS, Olk RJ, Burgess D, Gordon ME. Natural history of retinal pigment epithelial detachments in age-related macular degeneration. Ophthalmology. 1986;93(5):543-551.

6. Hartnett ME, Weiter JJ, Garsd A, Jalkh AE. Classification of retinal pigment epithelial detachments associated with drusen. Graefes Arch Clin Exp Ophthalmol. 1992;230(1):11-19.

7. [No authors listed]. Treatment of senile disciform macular degeneration: a single-blind randomised trial by argon laser photocoagulation. The Moorfields Macular Study Group. Br J Ophthalmol. 1982;66(12):745-753.

8. Baumal CR, Reichel E, Duker JS, Wong J, Puliafito CA. Indocyanine green hyperfluorescence associated with serous retinal pigment epithelial detachment in age-related macular degeneration. Ophthalmology. 1997;104(5):761-769.
9. Slakter JS, Yannuzzi LA, Sorenson JA, Guyer DR, Ho AC, Orlock DA A pilot study of indocyanine green videoangiography-guided laser photocoagulation of occult choroidal neovascularization in age-related macular degeneration. Arch Ophthalmol. 1994;112(4):465-472.

10. Axer-Siegel R, Ehrlich R, Avisar I, et al. Combined photodynamic therapy and intravitreal triamcinolone acetonide injection for neovascular age-related macular degeneration with pigment epithelium detachment. Ophthalmic Surg Lasers Imaging. 2006;37(6):455-461.

11. Axer-Siegel R, Ehrlich R, Rosenblatt I, et al. Photodynamic therapy for occult choroidal neovascularization with pigment epithelium detachment in age-related macular degeneration. Arch Ophthalmol. 2004;122(4):453-459.

12. Wygnanski-Jaffe T, Desatnik H, Alhalel A, et al. ICG angiographyguided photodynamic therapy for large pigment epithelial detachments in age-related macular degeneration. Ophthalmic Surg Lasers Imaging. 2006;37(5):358-363.

13. Cheung CM, Wong TY. Ranibizumab and bevacizumab for AMD. N Engl J Med. 2011;365(23):2237; author reply 2237.

14. Gragoudas ES, Adamis AP, Cunningham ET Jr, Feinsod M, Guyer DR. Pegaptanib for neovascular age-related macular degeneration. $N$ Engl J Med. 2004;351(27):2805-2816.

15. Rosenfeld PJ, Brown DM, Heier JS, et al. Ranibizumab for neovascular age-related macular degeneration. $N$ Engl J Med. 2006;355(14): 1419-1431.

16. Lalwani GA, Rosenfeld PJ, Fung AE, et al. A variable-dosing regimen with intravitreal ranibizumab for neovascular age-related macular degeneration: year 2 of the PrONTO Study. Am J Ophthalmol. 2009; 148(1):43-58. e41.

17. Ach T, Hoeh AE, Ruppenstein M, Kretz FT, Dithmar S. Intravitreal bevacizumab in vascular pigment epithelium detachment as a result of subfoveal occult choroidal neovascularization in age-related macular degeneration. Retina. 2010;30(9):1420-1425.

18. Arias L. Treatment of retinal pigment epithelial detachment with antiangiogenic therapy. Clin Ophthalmol. 2010;4:369-374.

19. Chen E, Kaiser RS, Vander JF. Intravitreal bevacizumab for refractory pigment epithelial detachment with occult choroidal neovascularization in age-related macular degeneration. Retina. 2007;27(4): $445-450$.

20. Introini U, Torres Gimeno A, Scotti F, Setaccioli M, Giatsidis S, Bandello F. Vascularized retinal pigment epithelial detachment in age-related macular degeneration: treatment and RPE tear incidence. Graefes Arch Clin Exp Ophthalmol. 2012;250(9):1283-1292.

21. Arora S, McKibbin M. One-year outcome after intravitreal ranibizumab for large, serous pigment epithelial detachment secondary to age-related macular degeneration. Eye (Lond). 2011;25(8):1034-1038.

22. Inoue M, Arakawa A, Yamane S, Kadonosono K. Variable Response of Vascularized Pigment Epithelial Detachments to Ranibizumab Based on Lesion Subtypes, Including Polypoidal Choroidal Vasculopathy. Retina. 2013;33(5):990-997.

\section{Publish your work in this journal}

Drug Design, Development and Therapy is an international, peerreviewed open-access journal that spans the spectrum of drug design and development through to clinical applications. Clinical outcomes, patient safety, and programs for the development and effective, safe, and sustained use of medicines are a feature of the journal, which

\section{Dovepress}

has also been accepted for indexing on PubMed Central. The manuscript management system is completely online and includes a very quick and fair peer-review system, which is all easy to use. Visit http://www.dovepress.com/testimonials.php to read real quotes from published authors. 